\title{
Systemic Therapy for Women with ErbB2-Positive Breast Cancer: New Options, New Challenges
}

\author{
Christian Jackisch $^{\mathrm{a}}$ Michael Untch ${ }^{\mathrm{b}}$ \\ ${ }^{a}$ Department of Obstetrics and Gynecology and Breast Cancer Center, Klinikum Offenbach, \\ ${ }^{\mathrm{b}}$ Frauenklinik/Interdisziplinäres Brustzentrum HELIOS-Klinikum Berlin-Buch, Germany
}

Advances in understanding the biology of breast cancer have led to the classification of tumors based upon their molecular features. With the advent of targeted therapies for both early and metastatic breast cancer (MBC), treatments are increasingly tailored towards the underlying individual tumor biology. Endocrine therapy for hormone receptor(HR)-positive tumors as well as compounds directed towards the inhibition of the ErbB2 (HER2) receptor are the two main advances in targeted therapy for women presenting with breast cancer, and have led to great strides in the understanding and treatment of this heterogeneous tumor entity. ErbB2 is a transmembrane receptor with tyrosine kinase activity, and is the most important growth factor within a family of related proteins in breast cancer. The ErbB group is composed of the 4 members ErbB1, ErbB2, ErbB3, and ErbB4. Activation of the ErbB autocrine growth pathway is a common mechanism for autonomous, dysregulated tumor cell proliferation and differentiation as well as angiogenesis, invasion, metastasis, and inhibition of apoptosis in the majority of human epithelial cancers. Dysfunctional ErbB2 signaling networks are reportedly present in a cohort of breast carcinomas with poor prognosis. In this respect, the ErbB2 pathway has become an attractive therapeutic target.

Based upon the success of the monoclonal antibody trastuzumab as the first ErbB2-targeted approach to reach the clinic, other strategies to block the ErbB2 signaling pathway have been developed, including lapatinib. Lapatinib is an innovative oral, small-molecule tyrosine kinase inhibitor (TKI) with a unique dual-TKI inhibitory activity against both ErbB1 and ErbB2 which are often overexpressed in breast cancer cells. The dual blockade leads to the downregulation of both the MAPK and PI3K signaling cascades responsible for cell proliferation and survival. Lapatinib has shown to be an extremely effective drug in the clinical setting. Combination therapy with lapatinib and capecitabine offers the only approved treatment for women with ErbB2-positive MBC, who have progressed after previous treatment with anthracyclines, taxanes, and in the metastatic setting with trastuzumab. The addition of lapatinib to capecitabine significantly prolongs time to progression, is associated with a trend toward improved overall survival, and offers the convenience of oral administration [1, 2]. Consistent with earlier results suggesting that lapatinib crosses the blood-brain barrier [3], the incidence of symptomatic brain metastases as first site of progression was significantly reduced when lapatinib was added to capecitabine therapy [2]. Due to the fact that ErbB2-overexpressing breast tumors are associated with an increased risk of developing brain metastases, these findings may have significant clinical relevance. Furthermore, there are interesting data suggesting that lapatinib is active in the treatment of women with ErbB2- and HR-co-expressing disease, enhancing endocrine responsiveness and/or restoring endocrine sensitivity. As demonstrated in the multicenter phase III EGF30008 trial, the combined targeted strategy with letrozole and lapatinib significantly increased progression-free survival and clinical benefit rates in postmenopausal women [4]. That strategy could pose as an appropriate approach for patients who are not in an acutely life-threatening situation. In addition, there is a growing body of evidence that lapatinib may be a candidate to overcome trastuzumab resistance due to its different mechanism of action. For instance, cells stably expressing the truncated receptor p95ErbB2 were resistant to trastuzumab but remained sensitive to the antiproliferative effects of lapatinib, both in vitro and in vivo [5]. In addition, loss of PTEN did not affect the induction of tumor cell apoptosis by lapatinib in different cell lines [6], suggesting that lapatinib seems to exert its antitumor activity in ErbB2overexpressing breast cancers in a PTEN-independent manner. This is the scientific rationale for using lapatinib + capecitabine for MBC patients as 2nd-line treatment after progressing on a 1st-line trastuzumab-containing regimen, and a good

\section{KARGER}

Fax +497614520714

Information@Karger.de

www.karger.com
(C) 2010 S. Karger GmbH, Freiburg

Accessible online at:

www.karger.com/brc
Prof. Dr. Christian Jackisch

Klinikum Offenbach

Frauenklinik

Starkenburgring 66, 63069 Offenbach, Germany

Tel. +49 69 8405-3850, Fax -4456

christian.jackisch@klinikum-offenbach.de 
example of how results from a translational research program contribute to a change in clinical practice. Lapatinib shows an acceptable safety profile. The most common adverse events are diarrhea, nausea and rash. Early intervention in case of diarrhea is recommended $[1,2,7]$. The incidence of cardiotoxicity seems to be low.

Various lapatinib combinations with agents such as paclitaxel, vinorelbine, or other molecular targeted agents are currently being investigated in clinical trials. Lapatinib is also studied in the treatment of early ErbB2-overexpressing breast cancer. Large adjuvant randomized trials using lapatinib following chemotherapy and surgery like the TEACH and ALTTO studies are ongoing. The randomized, multicenter, open-label phase III Neo-ALTTO study aims at exploring lapatinib as a neoadjuvant treatment option in ErbB2-overexpressing breast cancer. The Geparquinto study recruits patients to neoadjuvant treatment with anthracyclines and taxanes and compare the addition of trastuzumab to lapatinib in terms of pathologic complete remission. Results from these trials are eagerly awaited to fully define the role of lapatinib in the adjuvant and neo-adjuvant setting.

\section{Disclosure Statement}

The authors were speakers and contributors to the meeting 'ErbB2 (HER2)-positives Mammakarzinom; 2. Münchner Brustkrebs-Symposium Update 2009', 23/24 October 2009 in Munich, sponsored by GlaxoSmithKline.

\section{References}

$\checkmark 1$ Geyer CE, Forster J, Lindquist D, et al.: Lapatinib plus capecitabine for HER-2-positive advanced breast cancer. New Engl J Med 2006;355:2733-2743.

2 Cameron D, Casey M, Press M, et al.: A phase III randomized comparison of lapatinib plus capecitabine versus capecitabine alone in women with advanced breast cancer that has progressed on trastuzumab: updated efficacy and biomarker analyses. Breast Cancer Res Treat 2008;112:533-534.

$\checkmark 3$ Lin NU, Winer EP: Multicenter phase II study of lapatinib in patients with brain metastases from HER2-positive Breast Cancer. Clin Cancer Res 2009;15:1452-1459.
4 Johnston S, Pippen J Jr, Pivot X, et al.: Lapatinib combined with letrozole versus letrozole and placebo as first-line therapy for postmenopausal hormone receptor-positive metastatic breast cancer. J Clin Oncol 2009:27:5538-5546.

5 Scaltriti M, Rojo F, Ocaña A, et al.: Expression of p95HER2, a truncated form of the HER2 receptor, and response to anti-HER2 therapies in breast cancer. J Natl Cancer Inst 2007;99:628-638.
6 Xia W, Intisar H, Leihua L, et al.: Lapatinib antitumor activity is not dependent upon phosphatase and tensin homologue deleted on chromosome 10 in ErbB2-overexperssing breast cancers: Cancer Res 2007;67:1170-1175.

7 Benson AB, Ajani JA, Catalano RB: Recommended guidelines for treatment of cancer treatmentinduced diarrhea. J Clin Oncol 2004;22:2918-2926. 